\title{
Colgajo braquial lateral de flujo inverso para cobertura de la región posterior del codo, tras exéresis de sarcoma de partes blandas
}

\author{
D. Montaner', I. Pérez-Correa², A. Pérez-Blasco', J. Mallent ${ }^{3}$ \\ 'Servicio de Cirugía Ortopédica y Traumatología. Hospital Universitario Doctor Peset. Valencia. España. ${ }^{2}$ Servicio de Cirugía \\ Ortopédica y Traumatología. Hospital Francesc de Borja. Gandía Valencia. España. ${ }^{3 S}$ ervicio de Cirugía Plástica. Hospital de \\ la Ribera. Alzira Valencia. España.
}

\begin{abstract}
Resumen: La reconstrucción de los grandes defectos del codo, es un objetivo difícil, por técnicas convencionales, que aún se complica más cuando tenemos que aportar tejidos con la suficiente elasticidad y resistencia para que nos posibiliten una rehabilitación precoz, y además poder resistir una terapia externa abrasiva como la radioterapia, en el caso de tumores malignos. Presentamos la evolución a largo plazo de un paciente afecto de un Sarcoma de Células Foliculares Dendríticas (TCFD), que afectaba a la parte postero-cubital del codo, que tras su extirpación en bloque, se reconstruyó con un colgajo pediculado braquial lateral de flujo inverso. Exponemos caso clínico y técnica quirúrgica.

El uso del colgajo braquial lateral de flujo inverso, como método de reconstrucción en los defectos postquirúrgicos resultantes de la ablación tumoral, tiene la ventaja de ser una técnica fácil y reproducible con mínima morbilidad de la zona donante, ya que no sacrifica arterias ni músculos principales, no precisa microcirugía, por lo que se convierte en una buena alternativa a colgajos libres, y nos aporta un excelente resultado estético de color, grosor y fundamentalmente, textura, que favorecerán una rehabilitación precoz de la extremidad.
\end{abstract}

Palabras claves: Codo, células dendríticas, colgajo fascio-cutáneo, sarcoma.

\begin{abstract}
Introduction: The reconstruction of large defects of the elbow, is a difficult target for conventional techniques, which further complicated when we have to provide enough tissue elasticity, and resistance to enable us early rehabilitation, and also able to withstand external therapy abrasive as radiotherapy in malignant tumors.

We report the long-term outcome of a patient with a follicular dendritic cells sarcoma (FDCS), affecting the posterior ulnar elbow, which after en bloc resection, was reconstructed with a lateral brachial flap reverse flow. We present a case report and surgical technique.

The use of lateral brachial flap reverse flow, as a method of reconstruction in post-surgical defects resulting from tumor ablation has the advantage of being easy and reproducible technique with minimal donor site morbidity, and that does not sacrifice arteries or major muscles, not precise microsurgery, so it becomes a good alternative to free flaps, and fundamentally gives us an excellent result of color, thickness and texture, that encourage early rehabilitation of the limb.
\end{abstract}

Key words: Elbow, dendritic cells, fascio-cutaneous flap, sarcoma.

El tumor de células foliculares dendríticas (TCFD) es una neoplasia infrecuente cuyo origen está en las células presentadoras de antígeno del sistema inmune accesorio, de origen mesenquimal. Solo un tercio de los casos presentan una localización primaria extraganglionar, afectando fundamentalmente a cavidad oral, retroperitoneo, mediastino, pulmón, páncreas, hígado, bazo, tiroides, mama y región de cabeza y cuello. La localización cutánea es excepcional y no hemos localizado referencias al respecto en la bibliografía. Describimos el caso y planteamos una reconstrucción mediante un colgajo fasciocutáneo pediculado braquial lateral de flujo inverso. Se trata de una cobertura poco utilizada pero que sin embargo presenta una mínima morbilidad en la zona donante.

\section{CASO CLÍNICO}

Paciente varón de 62 años de edad que presenta una tumoración no dolorosa, multinodulada, adherida a planos profundos, en el tercio proximal de la región cubital del antebrazo izquierdo de dos años de evolución. Había sido diagnosticado previamente como
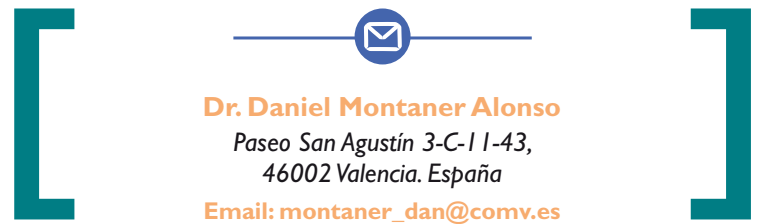


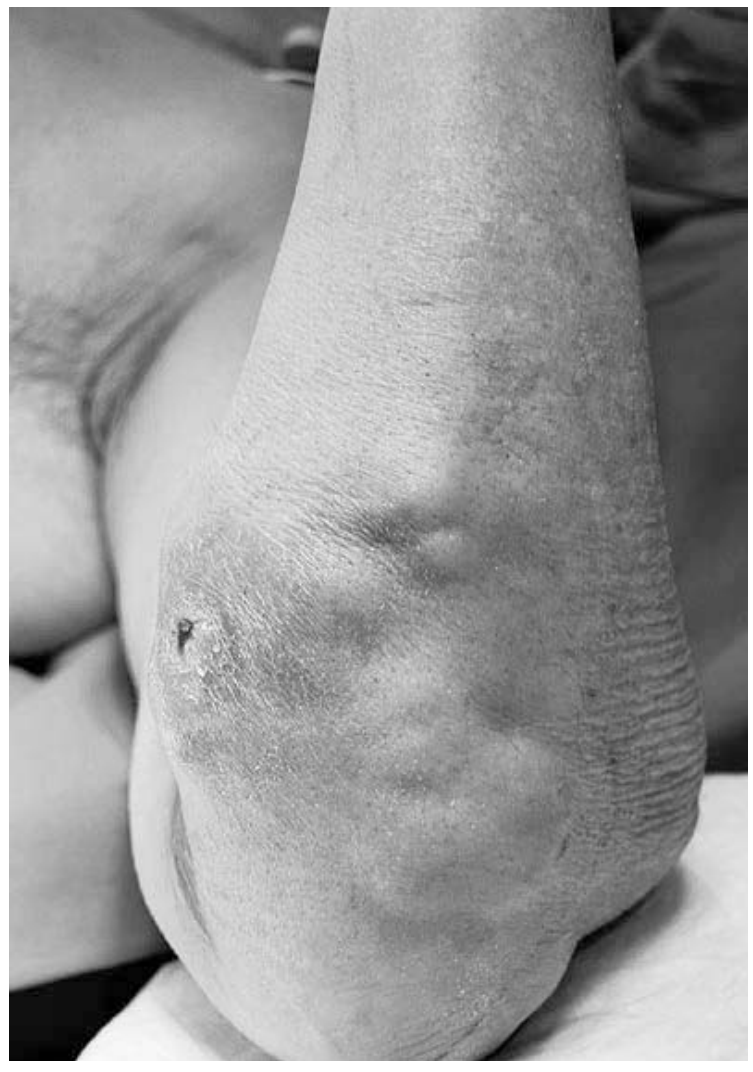

Figura. I. Imagen macroscópica tumoral: tumoración de 2 años de evolución sobre región cubital tercio proximal antebrazo izquierdo. Se aprecia la cicatriz resultante de la biopsia incisional.

lipomatosis y es atendido en servicio de cirugía ambulatorio, donde se procede al diagnóstico @ Figura I.

En el estudio mediante Resonancia Magnética se apreciaban tres masas de bordes bien definidos en tejido celular subcutáneo que no infiltraban musculatura, con heterogenicidad de señal en T2, e hiperintensidad de señal central de la lesión en la secuencia STIR, siendo estas imágenes compatibles con fascitis nodular, fascitis pseudosarcomatosa o neurofibroma plexiforme (1) Figura 2.

El diagnóstico preoperatorio definitivo de tumor de células foliculares dendríticas se obtuvo de forma externa a nuestro centro por parte de servicio de cirugía ambulatoria, mediante biopsia cutánea incisional. Posteriormente fue remitido al Servicio de Cirugía Ortopédica de nuestro centro.

El estudio de Anatomía Patológica indicaba la existencia de infiltración de hipodermis y tejido celular subcutáneo por una proliferación de células grandes,
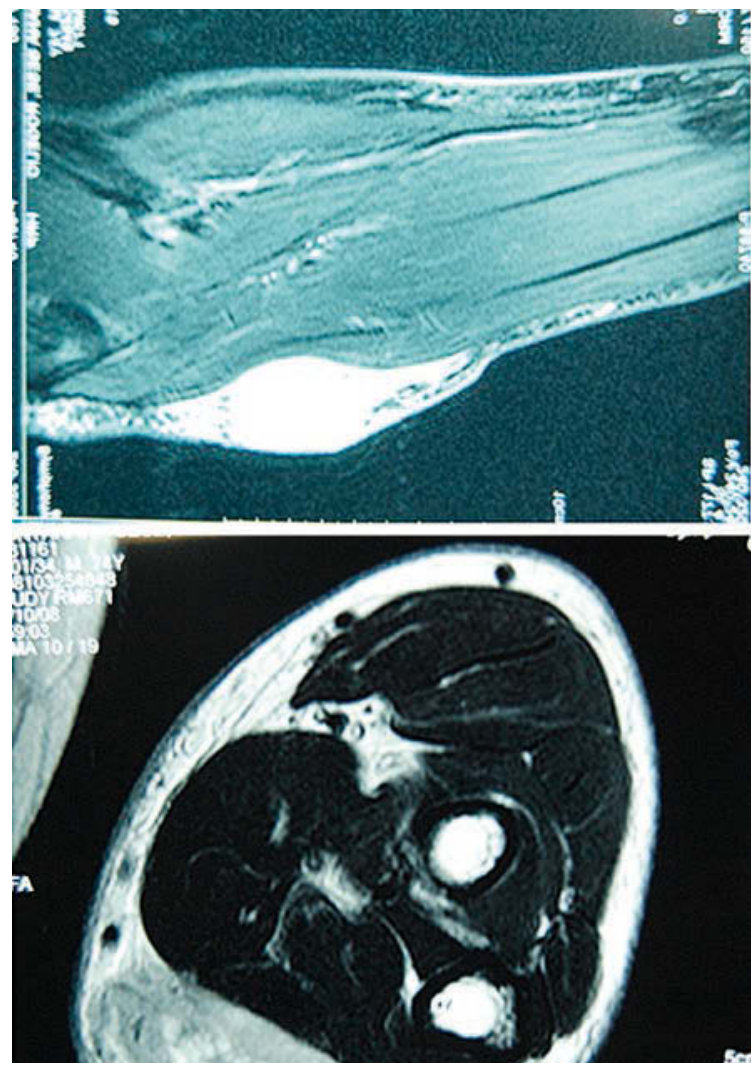

Figura 2. RNM: tres masas de $6 \times 4 \mathrm{~cm}$., bordes bien definidos, afectación de TCS, aparentemente no existe infiltración de la musculatura. Compatible con fascitis nodular, fascitis pseudosarcomatosa o neurofibroma plexiforme.

epitelioides y focalmente fusiformes, de amplio citoplasma y límites imprecisos, que se dispone a modo de nódulos entremezclados con linfocitos maduros. Los núcleos son atípicos y existen frecuentes mitosis. No hay necrosis tumoral. En el estudio inmunohistoquímico se evidencia positividad para vimentina, CD68, CD2I (marcador de células foliculares dentríticas), y siendo negativos los marcadores para citoqueratinas, HMB-45, S I00, CD34, marcadores neuroendocrinos, musculares, linfoides, mieloperoxidasa, lisozima y CDIa, característicos de otros tumores como carcinomas, melanoma, linfoma, sarcoma epitelioide, granulocítico y neoplasias de diferenciación muscular, neural, adipocítica y neuroendocrina. Por tanto, se estableció el diagnóstico de tumor maligno de estirpe mesenquimal y fenotipo epitelioide inmunohistoquímicamente positivo para CD68 y CD2I concordante con sarcoma de células foliculares dendríticas.

El estudio de extensión mediante Tomografía Axial Computarizada y Tomografía de Emisión de resultaron negativas, para la existencia de metástasis, por lo 

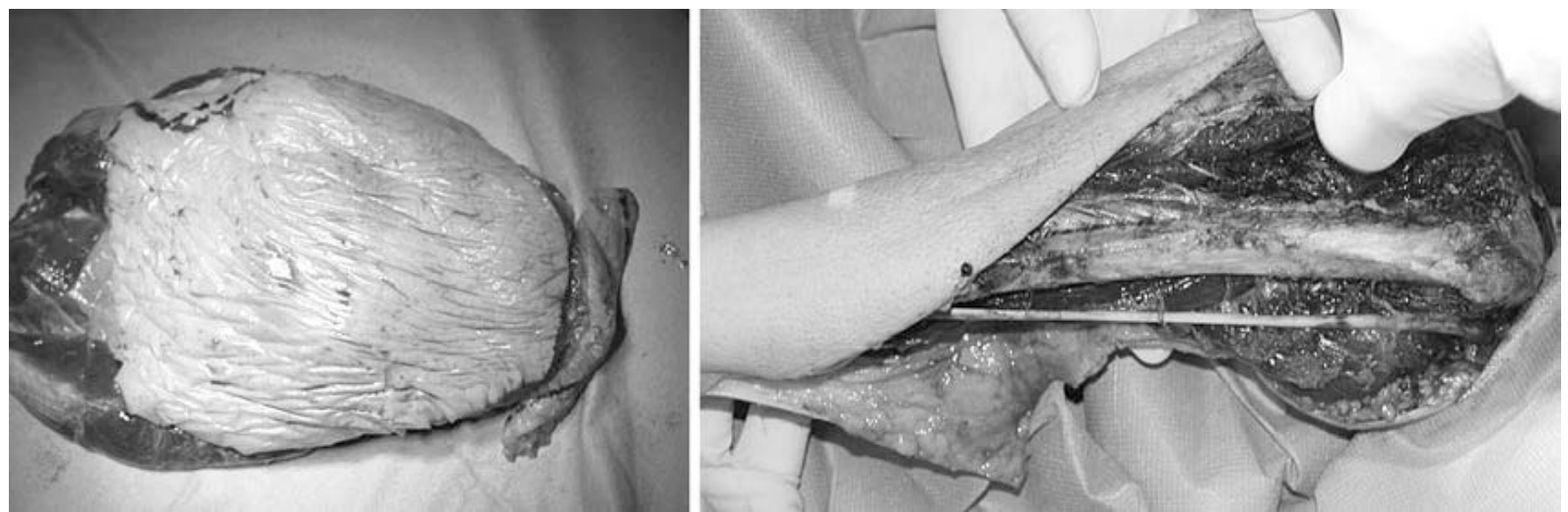

Figura 3. Resección en bloque de la masa tumoral con márgenes que incluyen musculatura pronato-flexora. Defecto creado tras la resección. Persistencia del nervio cubital y mediano.

que se planteó el tratamiento quirúrgico con intención curativa, mediante extirpación radical en bloque de la lesión cutánea y de las estructuras subyacentes afectadas hasta alcanzar márgenes libres de tumor mediante estudio anatomopatológico intraoperatorio.

En la cirugía, se extirpó en bloque con márgenes ampliados, la piel (supuestamente ya contaminada por la biopsia realizada), tejido celular subcutáneo y la musculatura pronatoflexora junto a la arteria Ulnar, la cual se encontraba dentro de los márgenes de seguridad de la pieza, pero se conservaron tanto en nervio Mediano como el Cubital, así como los músculos Flexor Policis Longus y Flexor Digitorum Profundus del segundo dedo (2) Figura 3.

Durante el mismo tiempo quirúrgico se llevó a cabo la reconstrucción de la zona afectada:

1. Los tendones flexores profundos de tercer, cuarto y quinto dedo se motorizaron mediante transposición del tendón del Extensor Carpi Radialis Longus, a través de la membrana interósea (2) Figura 4A.

2. La cobertura de la zona más distal de la diáfisis cubital se realizó mediante un colgajo muscular de Brachiorradialis e injerto de piel total de zona inguinal (1) Figura 4B.

3. Para la cobertura de la región olecraniana y proximal del cúbito, se utilizó un colgajo fasciocutáneo pediculado braquial lateral de flujo inverso. El defecto creado en la cara lateral del brazo, se cubrió con injerto mallado de piel de espesor parcial del muslo.
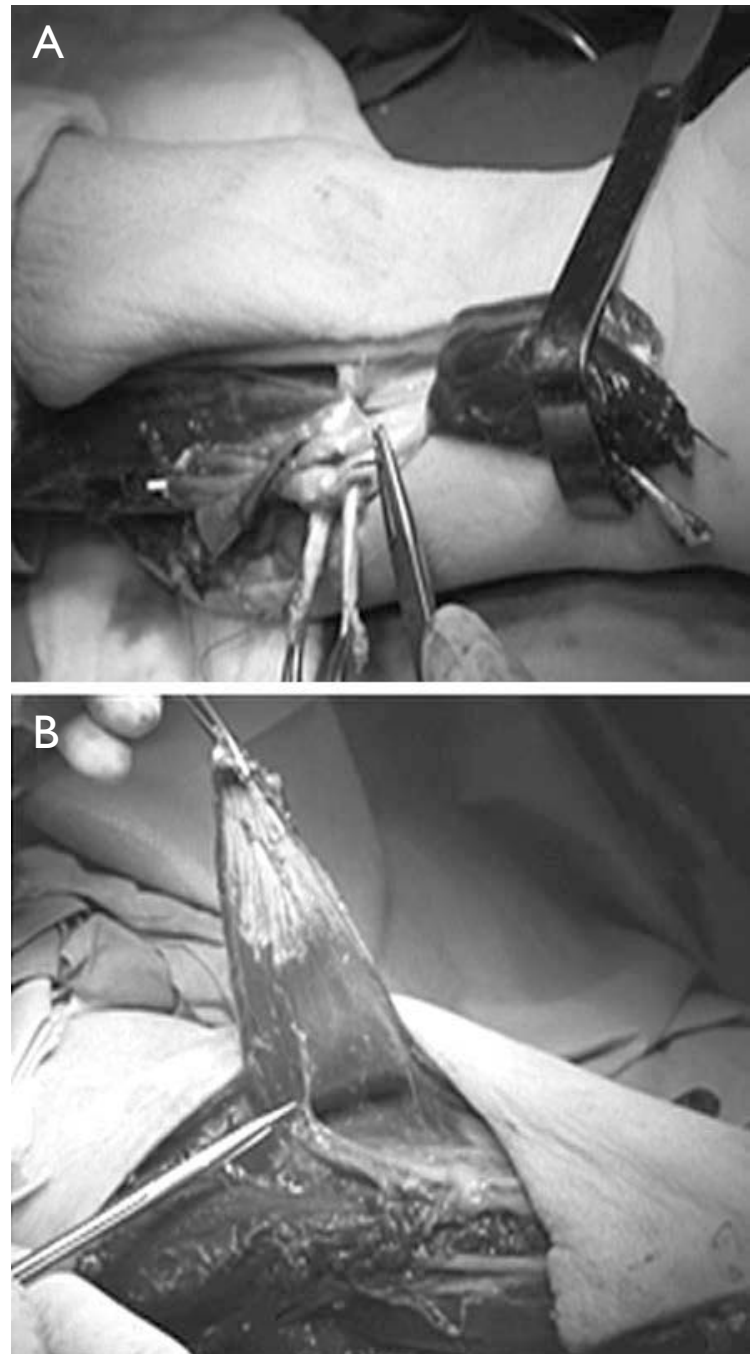

Figura 4. A. Transferencia de Primer radial a flexores profundos a través de membrana interósea. B. Músculo Brachiorradialis antes de su transposición hacia cara dorsal distal de antebrazo para cobertura del defecto cubital distal. 

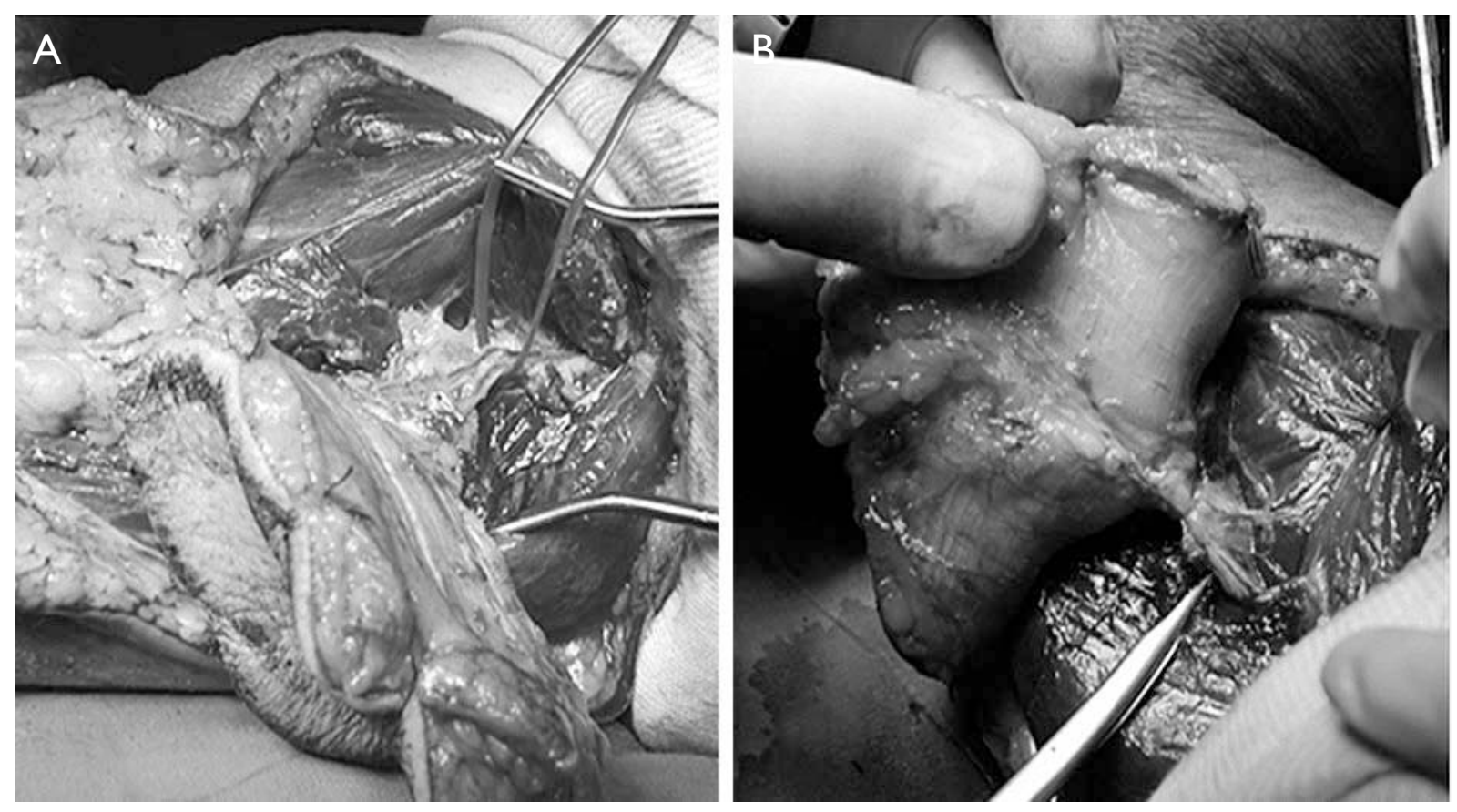

Figura. 5.A. Detalle del pedículo de la arteria humeral profunda, localizada, antes de su ligadura. B. Arterias perforantes el colgajo braquial cutáneo externo.

\section{TÉCNICA QUIRÚRGICA}

El colgajo braquial lateral es utilizado de forma habitual para coberturas proximales de la región del hombro, cuello y cara mediante la utilización de su pedículo principal dependiente de la rama posterior de la Arteria Humeral Profunda, que se anastomosa con la Arteria Recurrente Posterior de la Arteria Radial, y manteniendo la sensibilidad por el nervio cutáneo lateral inferior.

En nuestro caso, como la región a reparar y cubrir el la parte posterior y cubital del codo, debemos utilizar este colgajo con flujo invertido, es decir, utilizando la anastomosis arterial mencionada, y ligando en la zona proximal la Arteria Humeral Profunda.

Previamente a la cirugía debemos aseguramos de la presencia de esta arteria mediante la utilización de Eco-Doppler.Tras dibujar el colgajo, que se elabora a partir de la línea existente entre la parte distal al vértice del músculo deltoides, y en dirección al epicóndilo, comienza la disección de proximal a distal. Se debe llegar a exponer el Tríceps Braquial y el Supinador Largo, donde en su septo intermuscular encontraremos la Arteria Humeral Profunda, que ligaremos proximalmente, a partir de aquí seguiremos el pedículo fascial que procede de la Arteria Recurrente Radial (2) Figura 5A y 5B. Una vez disecada la parte externa separando el colgajo del músculo Brachialis, lo podemos liberar y se tuneliza subcutáneamente hasta alcanzar la zona del defecto!.

\section{RESULTADOS}

El estudio anatomopatológico de los bordes limítrofes informa de bordes libres. La pieza de resección correspondiente a la tumoración mide $18 \times 10 \times 5 \mathrm{~cm}$, con óvalo cutáneo de $18 \times 9 \mathrm{~cm}$ y sin alteraciones macroscópicas al corte. Se observa una tumoración con múltiples nódulos blanquecinos, con áreas de necrosis que mide $7 \times 6 \times 2,5 \mathrm{~cm}$ y se encuentra a $2 \mathrm{~cm}$ del borde quirúrgico de resección profundo. El diagnóstico es de sarcoma de células foliculares dendríticas con bordes quirúrgicos libres de tumor. Se observa intensa necrosis y llega a fascia sin infiltrarla. Se considera de alto grado histológico.

Posteriormente el paciente recibe radioterapia adyuvante a 60 Gy, durante 5 semanas. El colgajo de cobertura del codo no sufrió alteraciones durante el tratamiento adyuvante, a excepción del eritema local subsidiario a la radiación, pero no se originaron otras alteraciones dérmicas. 

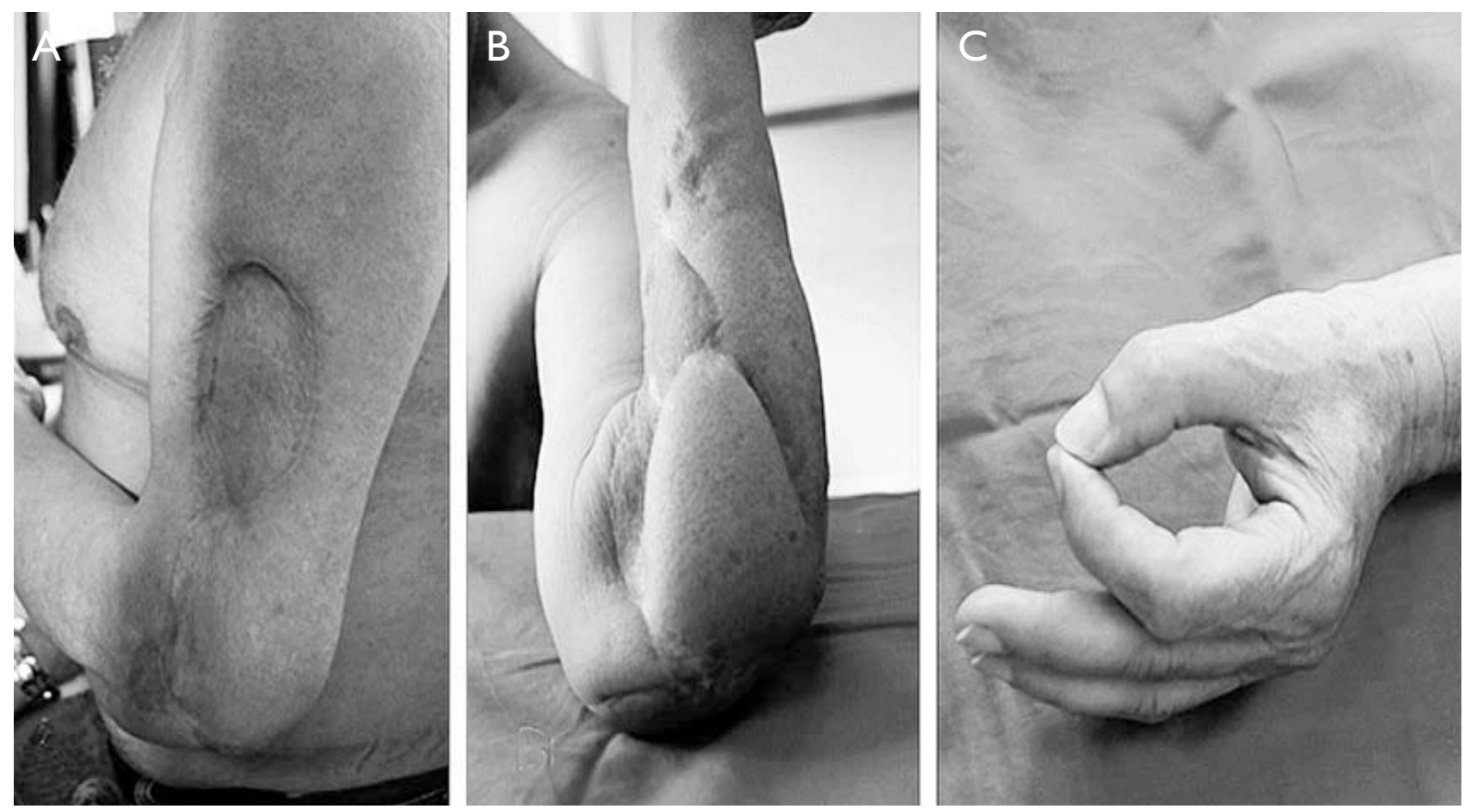

Figura 6.A y B. Tras 5 años, estado actual del defecto creado, aspecto del colgajo, y movilidad del codo. C. Pinza digital resultante tras las transposiciones tendinosas realizadas..

Actualmente han transcurrido 5 años tras el tratamiento, y los estudios de RM y TAC de codo y antebrazo, indican signos de osteopenia marcada en radio y cúbito afectando también a las corticales del hueso. Esta osteopenia puede ser debida a la radioterapia, y no se ven captaciones ni alteraciones que sugieran recidiva tumoral.

Desde el punto de vista de la clínica, la cobertura cutánea es adecuada, con una mínima morbilidad en la zona donante, que ha quedado correctamente epitelizada por el injerto libre y la recuperación funcional también es satisfactoria, con correcta flexo-extensión del codo, una adecuada movilidad de muñeca y dedos que le permiten agarrar objetos si bien con una flexión incompleta de los dedos, y la realización de pinza (a) Figura 6A, 6B y 6C.

\section{DISCUSIÓN}

Los sarcomas son un grupo heterogéneo de tumores poco frecuentes que se originan predominantemente a partir del mesodermo embrionario. Se presentan más comúnmente como una masa asintomática localizada en una extremidad (59\% casos), y es la biopsia la mejor técnica para su diagnóstico².
Los Sarcomas de Células Foliculares Dendríticas (TCFD) fueron descritos por Monda, Warnke y Rosai en 1986. Se clasificaron previamente como linfomas, sarcomas o tumores histiocíticos. ${ }^{3}$. Las neoplasias de células histiocitarias y dendríticas derivan del sistema mononuclear fagocitario y células accesorias, las cuales desempeñan su principal papel en el procesamiento y presentación de antígenos a los linfocitos. Las células dendríticas se distribuyen en muchos tejidos del organismo. A las ubicadas en los centros foliculares linfoides, se las denomina células dendríticas foliculares. Dichas células presentan inmunotinción para CD21, CD23 y CD35. Solo un tercio de los casos presentan una localización primaria extraganglionar, afectando fundamentalmente a cavidad oral, retroperitoneo, mediastino, pulmón, páncreas, hígado, bazo, tiroides, mama y región de cabeza y cuello ${ }^{4,5}$. La localización cutánea es excepcional y no hemos localizado referencias al respecto en la bibliografía.

La edad de presentación media es de 45 años y se distribuyen igualmente entre ambos sexos, no existiendo diferencias en estos aspectos entre los de localización ganglionar y extraganglionar. Se trata de tumores bien delimitados, ocasionalmente encapsulados, con una malignidad intermedia según la escala de la organización mundial de la salud. Producen metástasis en el $25 \%$ de los casos y presentan una mortalidad del 
10-20\%. La tasa de recidiva local es alta, produciéndose hasta en un 40-50\% de los casos.

Los sarcomas de intermedio y alto grado de la región del codo precisan de un tratamiento quirúrgico agresivo. En 1985, el National Institutes of Health formuló una declaración de consenso, recomendando una cirugía de preservación del miembro en la mayoría de los pacientes con sarcomas de las extremidades mediante una escisión ampliada. El objetivo era resecar el tumor con un margen de $2 \mathrm{~cm}$. de tejido blando circundante normal. Desde que se aplican estos criterios, el confort de los pacientes es mucho mayor, según el grado de función propuesto por Enneking, y a dejado la cirugía de amputación de la extremidad relegada a un tratamiento, digamos "in extremis". Ahora bien, tendremos que tener en cuenta en nuestra programación prequirúrgica la necesidad de sacrificar estructuras nobles, y por tanto planear una correcta reconstrucción lo más funcional posible así como una correcta cobertura del defecto creado.

La necesidad de sacrificar grandes vasos de la extremidad sin necesidad de reconstrucción posterior está descrita, y además de todos es conocido que las anastomosis distales entre las arterias Radial y Cubital hacen posible la subsistencia de la extremidad con una sola de ellas. Por otra parte, sí es importante que el aporte de tejido vascularizado para el cierre de los defectos sea de la suficiente calidad y elasticidad como para soportar los tratamientos adyuvantes postquirúrgicos necesarios para el tratamiento del sarcoma, como es la radioterapia, ya que la retracción que sufrirá la piel por la radiación térmica podría ser causa de diástasis y necrosis ${ }^{6}$. De todos son conocidos los efectos secundarios a más de un año, incluso a largo plazo, de la radioterapia postquirúrgica, y que fundamentalmente se trata de fibrosis, necrosis, edema, incluso fracturas y contracturas, y que todas ellas afectan a la función.

La naturaleza de los tejidos funcionales, que incluyen los grandes vasos, nervios y tendones, con la pobreza de partes blandas que lo recubren, con una dermis delgada y escasa grasa subcutánea, aumentan la dificultad de una amplia escisión en bloque de tumores del codo. Además, la dificultad técnica del procedimiento y la morbilidad asociada funcional se ve incrementada por la necesidad de lograr unos márgenes adecuados de resección $n^{7}$. Por este motivo necesitamos técnicas de rescate como son la utilización de grandes colgajos libres, como los de Latissimus Dorsi ${ }^{8}$. Sin embargo, en los casos en los que no existe una afectación articular o en los que el sacrificio de estructuras nobles o musculares no ha de ser tal que incapacite gravemente la función, podemos utilizar técnicas de cobertura locales. Estas técnicas, como son los colgajos de transferencia pediculados, son fácilmente reproducibles, y además debemos tener en cuenta que los colgajos libres requieren más experiencia y también puede fallar en algunas circunstancias. Por tanto, una técnica ideal, fácil y fiable a realizar en una sola etapa y cuyo procedimiento nos proporcione resultados predecibles quirúrgicos con respecto a la función del codo y la estética, es la utilización del colgajo lateral del brazo con flujo inverso, propuesto por Song en 1982, y posteriormente perfeccionado por Katsaros en 1984, quien mejoró la descripción anatómica y amplió sus aplicaciones clínicas $^{9}$. Posteriormente existen diversos trabajos que avalan la calidad, y capacidad de reproducción del método, tal y como lo hemos descrito anteriormente, y que en parte es debido al elevado número de perforantes que existen en los territorios arteriales de la cara lateral del brazo, y que de una manera constante se encuentran repartidas entre el septum muscular y procedentes de la Arteria Circunfleja Posterior en un número de 5 ○ 6, y con un calibre de entre 0,6 y 1,2 mm.

\section{CONCLUSIONES}

El uso del colgajo braquial lateral de flujo inverso, como método de reconstrucción en los defectos postquirúrgicos resultantes de la ablación tumoral, tiene las siguientes ventajas:

- Técnica fácil y reproducible con mínima morbilidad zona donante, ya que no sacrifica arterias ni músculos principales.

- No precisa microcirugía, por lo que se convierte en una buena alternativa a colgajos libres.

Excelente resultado estético: color, grosor y fundamentalmente textura, que favorecerán una rehabilitación precoz de la extremidad.

\section{CONFLICTOS DE INTERESES}

Los autores declaran no tener conflictos de intereses.

\section{BIBLIOGRAFÍA}

I. Masquelet AC, Gilbert A. Cormier JN, Pollock RE. Soft Tissue Sarcomas. CA-Cancer J Clin. 2004:54:94-109. 
2. Kairouz S, Hashash J, Kabbara W, McHayleh W, Tabbara IA. Dendritic cell neoplasms: an overview. Am J Hematol. 2007;82( I 0):924-8.

3. Camarasa-Lillo N, Rosellón-Sastre E, PalmeiroUriach A, García-Cosío-Piqueras M, Santón-Roldán A, Beiras A, Forteza-Vila J. Sarcoma histiocítico con rasgos inmunohistoquímicos y ultraestructurales de células dendríticas interdigitantes. Rev Esp Patol. 2008;4 I (3):237-4I.

4. Youens KE, Waugh MS. Extranodal follicular dendritic cell sarcoma. Arch Pathol Lab Med. 2008; | 32( I0): | 683-7.

5. Jorge-Buys DL, Lastra-Camacho G, Campos-Martínez J, Romero-Guadarrama M, Ortiz-Hidalgo C. Sarcoma de células dendríticas foliculares de localización ganglionar y extraganglionar. Estudio clinicopatológico e inmunohistoquímico de cinco casos. Cir Ciruj. 2008;76: |45-52.

6. Johansen R, Nielsen $\bigcirc$, Keller J. Functional outcome in sarcomas treated with limb-salvage surgery or amputation. Sarcoma. 1998;2;19-23.

7. Schwab JH; Healey JH; Athanasian EA. Wide en bloc extra-articular excision of the elbow for sarcoma with complex reconstruction. J Bone Joint Surg Br. 2008;90B(I):78-83.

8. Katsaros J, Schusterman M, Beppu M, Banis JC Jr, Acland RD. The lateral upper arm flap: anatomy and clinical applications. Ann Plast Surg. 1984; | 2(6):489-500.

9. Hwang K, Lee WJ, Jung CY, Chung $\Vdash H$. Cutaneous perforators of the upper arm and clinical applications. J Reconstr Microsurg. 2005;2I (7):463-9. 